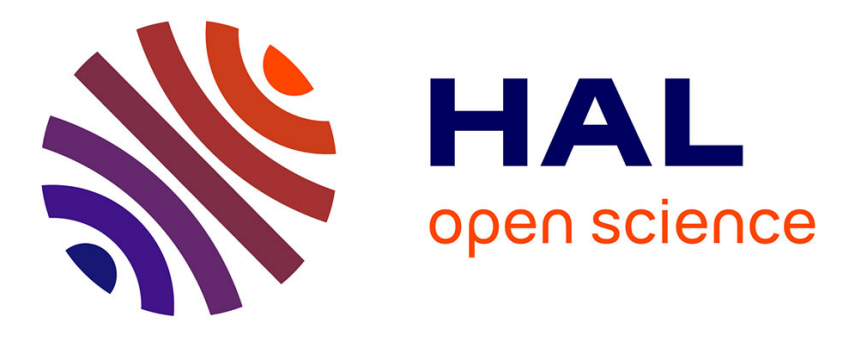

\title{
Optimal design of tensegrity mechanisms used in a bird neck model
}

\author{
Matthieu Furet, Anders van Riesen, Christine Chevallereau, Philippe Wenger
}

\section{To cite this version:}

Matthieu Furet, Anders van Riesen, Christine Chevallereau, Philippe Wenger. Optimal design of tensegrity mechanisms used in a bird neck model. European Conference on Mechanism Science (EUCOMES'2018), Sep 2018, Aachen, Germany. pp.365-375, 10.1007/978-3-319-98020-1_43 . hal01989992

\section{HAL Id: hal-01989992 \\ https://hal.science/hal-01989992}

Submitted on 22 Jan 2019

HAL is a multi-disciplinary open access archive for the deposit and dissemination of scientific research documents, whether they are published or not. The documents may come from teaching and research institutions in France or abroad, or from public or private research centers.
L'archive ouverte pluridisciplinaire HAL, est destinée au dépôt et à la diffusion de documents scientifiques de niveau recherche, publiés ou non, émanant des établissements d'enseignement et de recherche français ou étrangers, des laboratoires publics ou privés. 


\title{
Optimal design of tensegrity mechanisms used in a bird neck model
}

\author{
Matthieu Furet ${ }^{1}$, Anders van Riesen ${ }^{1,2}$, Christine Chevallereau ${ }^{1}$, and Philippe \\ Wenger $^{1}$ \\ 1 Laboratoire des Sciences du Numérique de Nantes (LS2N), CNRS, Ecole centrale \\ de Nantes, 44321 Nantes, France \\ 2 University of Twente, 7500 AE Enschede, The Netherlands
}

\begin{abstract}
This paper deals with the optimal design of an antagonistically actuated X-shape Snelson tensegrity mechanism to be used in a preliminary bird neck model made of a series of cascaded such mechanisms. The mechanism, subject to its own weight and to the weight of the subsequent mechanisms, is designed to maximize its wrench feasible workspace under given maximal actuation forces. Moreover, the mechanism is constrained to stand in a prescribed rest configuration. The optimized parameters are the link lengths and the springs stiffness.
\end{abstract}

Keywords: Tensegrity, wrench feasible workspace, design, stiffness

\section{Introduction}

This work falls within the frame of the AVINECK project, a collaboration project with biologists, in which a robotic model of a bird neck shall be designed and built. Birds use their neck as an arm. It exhibits very interesting properties such as a high dexterity (e.g. the vulture can tear meat inside a carcass), a high dynamics (e.g. the woodpecker makes holes with high-frequency motions) or a high payload-to-weight ratio (e.g. the parrot can hang from a cage bar using its beak and thus carry its own weight). Contrary to muscular hydrostats (elephant trunks or cephalopod tentacles), bird necks have a skeletal spine like snakes, but contrary to the latter, bird necks do not lie on the ground. The concept of tensegrity has been chosen in this project as a general paradigm able to link the interests of biologists and roboticists. A tensegrity structure is made of compressive and tensile components held together in equilibrium [1], [2]. Tensegrity structures were first used in art [3] and have then been applied in civil engineering [4] and robotics [5], [6], [7], [15]. There are suitable to model muskuloskeleton structures where the bones are the compressive components and the muscles and tendons are the tensile elements [8]. A preliminary, planar bird neck robotic model is considered in this paper. This model is built upon stacking a series of Snelson's $\mathrm{X}$-shape mechanisms [2]. Although simplified because it is planar, this model goes beyond the only available bird neck model in the literature that uses a simple planar articulated linkage [13]. Snelson's X-shape mechanisms have been 
studied by a number of researchers, either as a single mechanism [5],[7],[9] or assembled in series [10],[11],[12]. In this paper, the mechanism is actuated with two lateral tendons threaded through the spring attachment points like in [12]. This mechanism differs from to the ones analyzed in [5],[7],[9],[10],[11] in that its upper link is a rigid bar, a choice that aims at keeping to one the mobility of each mechanism. This mechanism is a tensegrity mechanism of class 2 (2 compressive elements linked together [4]) and the neck model resulting from stacking several such mechansims if of class 3. Finally, the mechanism is supposed to operate in a vertical plane and is thus subject to gravity, unlike in [12], where the mechanism was used in a snake-like manipulator moving on the ground. The goal of this study is to provide a preliminary design scheme of the elementary tensegrity mechanisms that are to be cascaded in a preliminary bird neck model prototype.

\section{Description of the neck model and design strategy}

We would like to build a prototype composed of a number of stacked elementary mechanisms or segments where each segment represents a vertebra of a bird neck. The number of vertebrae depends on the bird specie (from 10 in the parrot to 26 in the swan [14]). The design aims at determining the optimal link lengths and springs stiffness for a maximal wrench feasible workspace under prescribed maximal actuation force constraints. Moreover, the mechanism is constrained to stand in a prescribed rest configuration, so that, when all the mechanisms are stacked together, the resulting model takes the characteristic S-shape rest posture observed in all bird necks [14], see Figure 1 .

The design of the full neck model is conducted in statics only, which makes it possible to reduce the design problem to the sequential design of each segment, starting with the last one. Each segment must carry the sub-chain made of the mechanisms stacked overhead and the head (only the head for the last segment). Accordingly, the segment under design is subject to a vertical force $F_{P}$ acting on a point $P$, where $P$ is the center of gravity of the aforementioned sub-chain and $F_{P}$ is its weight. Each segment is described in its base reference frame, defined such that its origin coincides with hinge $\mathrm{A}$ and the $\mathrm{x}$-axis is aligned with the base link 1 (Figure 2). The coordinates $\hat{x}_{P}, \hat{y}_{P}$ of $P$ are defined in a frame attached to the upper link 4 as in Figure 2. These coordinates are assumed known and their determination is out of the scope of this study as they rely on the determination of some critical configuration, which is not straightforward.

A single segment of the bird neck model consists of a symmetric four-bar mechanism with crossed links and two pre-tensioned springs, as shown in Figure 2. The four links are rigid, homogeneous and linear bars of mass $m_{i}, i=1 . .4$, jointed at A, B, C and D. The two crossed bars (resp. the upper and lower bar) are of equal length $L$ (resp. b). The two springs connect $\mathrm{A}$ and $\mathrm{D}$, and $\mathrm{B}$ and $\mathrm{C}$, respectively. Their free length is defined as $l_{0}=L-b$, which corresponds to the minimal length of $\mathrm{AD}$ and $\mathrm{BC}$ reached when the mechanism is in its flat singular configurations. Since the mechanism is constrained to operate out of its 
singularities (we impose $-\pi<\alpha<\pi$ ), $l_{0}=L-b$ ensures that the springs are always in tension.

The mechanism is actuated by tendons connected at D and $\mathrm{C}$ and threaded through the springs. The tendons are assumed infinitely stiff, hence input forces $F_{1}$ and $F_{2}$ are considered to act directly on $\mathrm{D}$ and $\mathrm{C}$, respectively. Since the tendons cannot push, $F_{1}$ and $F_{2}$ are always positive and they are bounded by $F_{\text {max }}$, which depends on the actuators used.

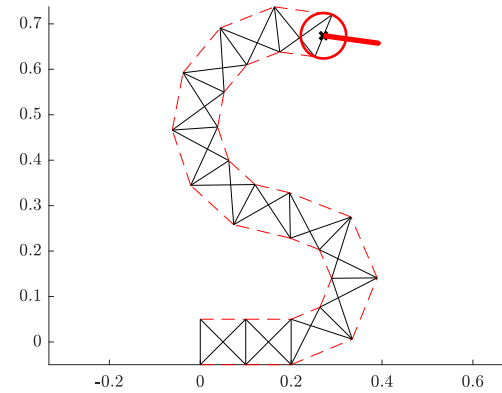

Fig. 1: Bird neck model at rest

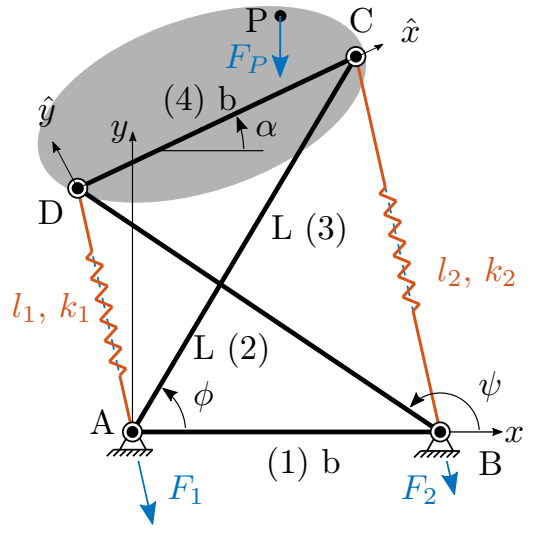

Fig. 2: Model of a single segment.

The rigid bars will be $3 \mathrm{D}$ printed with ABS material of circular cross section with a fixed diameter. Thus, $m_{2}=m_{3}=\rho_{m} L, m_{4}=m_{1}=\rho_{m} b$, where $\rho_{m}$ is the mass per unit length. With a cross section diameter fixed to $0.01 \mathrm{~m}, \rho_{m}=0.0825 \mathrm{~kg} / \mathrm{m}$. Two adjacent segments share the same bar, namely, the upper bar of the lower segment is the base bar of the upper segment.

The spring masses $m_{r i}, i=1,2$ cannot be neglected here as they turn out to be of the same order as the bar masses. In order to express $m_{r i}$ as a function of the stiffness $k_{i}$ and $L$, the two relations below are used:

$$
k_{i}=\frac{G d^{5}}{8 D^{3} l_{0}} \quad \text { (1) } \quad m_{r i}=\rho_{s} \frac{\pi^{2} D d l_{0}}{4}
$$

where $G$ and $\rho_{s}$ are the shear modulus and density, respectively; $D$ and $d$ are the spring and wire diameters, respectively and are linearly dependent: $D=\lambda d$. Combining (1) and (2), $m_{r i}$ can be expressed as $m_{r i}=\nu k_{i} l_{0}^{2}$, where $\nu$ is a parameter depending on the spring material and geometry. For a steel spring, choosing $\lambda=8$ and knowing that $l_{0}=L-b, m_{r i}=0.008 k_{i}(L-b)^{2}$.

Without loss of generality, the length of the base and upper bars are fixed to $b=0.1 \mathrm{~m}$ and the only length parameter to be optimized is $L$. For the optimization problem, finally, there are only three design parameters for each segment: $L, k_{1}$ and $k_{2}$.

\section{Segment modeling}

The orientation of the mechanism upper bar $\alpha$ is chosen to specify the configuration of each segment. The orientation of movable links 2 and 3 are defined by 
$\phi$ and $\psi$, respectively (Figure 2). The loop-closure constraint equation written in $A$ along $x$ and $y$ :

$$
\begin{array}{r}
b+L \cos (\psi)+b \cos (\alpha)-L \cos (\phi)=0 \\
L \sin (\psi)+b \sin (\alpha)-L \sin (\phi)=0
\end{array}
$$

allows one to write $\phi$ and $\psi$ as a function of $\alpha$ :

$$
\begin{aligned}
& \phi(\alpha)=2 \arctan \left[\frac{2 b L \sin (\alpha)+S}{\left(2 b^{2}+2 b L\right)[\cos (\alpha)+1]}\right] \\
& \psi(\alpha)=2 \arctan \left[\frac{-2 b L \sin (\alpha)-S}{\left(2 b^{2}-2 b L\right)[\cos (\alpha)+1]}\right]
\end{aligned}
$$

with $S=\sqrt{(-2 b L \sin (\alpha))^{2}+(-2 b L[\cos (\alpha)+1])^{2}-\left(2 b^{2}[\cos (\alpha)+1]\right)^{2}}$.

Using the cosine rule, the spring lengths can be expressed as follows:

$$
\begin{aligned}
& l_{1}=\sqrt{b^{2}+L^{2}+2 b L \cos (\psi)} \\
& l_{2}=\sqrt{b^{2}+L^{2}-2 b L \cos (\phi)}
\end{aligned}
$$

The static model of the segment can be obtained with its potential energy $V$ and the potential function $E_{e x}$ associated to the external wrench, assumed to be conservative [4]. Here, the external wrench represents the two actuation forces $F_{1}, F_{2}$ and the weight of the overhead sub-chain $F_{P}$. The segment is in equilibrium when:

$$
V^{\prime}=E_{e x}^{\prime}
$$

where the ' means the partial derivative with respect to $\alpha: \frac{\partial}{\partial \alpha}$. The potential energy $V$ is the sum of the potential energy $V_{b}$ associated with the bar masses and the potential energy $V_{s}$ associated with the springs stiffnesses and masses:

$$
\begin{aligned}
& V_{b}=\frac{g}{2}\left[m_{2} L \sin (\phi)+\left(m_{3}+2 m_{4}\right) L \sin (\psi)+m_{4} b \sin (\alpha)\right] \\
& V_{s}=\frac{1}{2} k_{1}\left(l_{1}-l_{0}\right)^{2}+\frac{1}{2} k_{2}\left(l_{2}-l_{0}\right)^{2}+\frac{g}{2}\left(m_{r 1} l_{1}+m_{r 2} l_{2}\right) \cos \left(\frac{\alpha}{2}\right)
\end{aligned}
$$

Note that for writing simplification purposes, the base of the segment under study is supposed to be horizontal here. For any other base orientation, a component of the gravity vector along $x$ must be considered, with the only technical consequence of making (7), (10c) and (10d) more lengthy.

The potential $E_{e x}$ is defined as:

$$
E_{e x}=-F_{1} l_{1}-F_{2} l_{2}-F_{p}\left(L \sin (\psi)+\hat{x}_{P} \sin (\alpha)+\hat{y}_{P} \cos (\alpha)\right)
$$

where $\hat{x}_{P}$ and $\hat{y}_{P}$ are the coordinates of $P$ in the frame attached to the upper link (Figure 2).

Reporting equations (8) and (7) into (6) and using (5), (4a) and (4b) yields the static equilibrium equation:

$$
G_{b}(\alpha, L)+G_{s}\left(\alpha, L, k_{1}, k_{2}\right)=Z_{1}(\alpha, L) F_{1}+Z_{2}(\alpha, L) F_{2}+Z_{p}(\alpha, L) F_{P}
$$


where:

$$
\begin{aligned}
Z_{i}(\alpha, L) & =-l_{i}^{\prime} \quad \text { for } i=1,2 \\
Z_{P}(\alpha, L) & =-L \cos (\psi) \psi^{\prime}-\hat{x}_{P} \cos (\alpha)+\hat{y}_{P} \sin (\alpha) \\
G_{b}(\alpha, L) & =\frac{g}{2}\left[m_{2} L \cos (\phi) \phi^{\prime}+\left(m_{3}+2 m_{4}\right) L \cos (\psi) \psi^{\prime}+m_{4} b \cos (\alpha)\right]
\end{aligned}
$$

$$
\begin{aligned}
G_{s}\left(\alpha, L, k_{1}, k_{2}\right) & =k_{1}\left[l_{1}^{\prime}\left(l_{1}-l_{0}\right)-\frac{g}{4} \nu l_{0}^{2} l_{1} \sin \left(\frac{\alpha}{2}\right)+\frac{g}{2} \nu l_{0}^{2} l_{1}^{\prime} \cos \left(\frac{\alpha}{2}\right)\right] \\
& +k_{2}\left[l_{2}^{\prime}\left(l_{2}-l_{0}\right)-\frac{g}{4} \nu l_{0}^{2} l_{2} \sin \left(\frac{\alpha}{2}\right)+\frac{g}{2} \nu l_{0}^{2} l_{2}^{\prime} \cos \left(\frac{\alpha}{2}\right)\right]
\end{aligned}
$$

The static equilibrium equations above depend only on $\alpha$ and on the design parameters $L, k_{1}$ and $k_{2}$. Indeed, $\psi$ and $\phi, l_{1}$ and $l_{2}$ can be expressed as a function of $\alpha$ using equations (5), (4), $l_{0}=L-b$ and all mass parameters can be expressed as a function of $L$ (remember also that $b$ was fixed to $0.1 \mathrm{~m}$ ).

To avoid lengthy derivations in the next section, the above equation is rewritten in a more compact way below:

$$
G_{s}\left(\alpha, L, k_{1}, k_{2}\right)=k_{1} X_{1}(\alpha, L)+k_{2} X_{2}(\alpha, L)
$$

Two situations will be considered for the design process: the neck is at rest and the two actuation forces are active. The behavior of the mechanism in these two situations are studied in the next sections.

\section{Behavior of the mechanism at rest}

The behavior at rest is defined from (9) as the behavior without actuation: $F_{1}=F_{2}=0 N$. Differentiating (9) with respect to $\alpha$ gives the mechanism stiffness $K_{\alpha}[5]$ :

$$
K_{\alpha}\left(\alpha, L, k_{1}, k_{2}\right)=G_{b}^{\prime}(\alpha, L)+G_{s}^{\prime}\left(\alpha, L, k_{1}, k_{2}\right)-Z_{p}^{\prime}(\alpha, L) F_{P}
$$

The design should allow the mechanism to have a stable equilibrium in a prescribed rest configuration $\alpha_{0}$. Accordingly, its stiffness $K_{\alpha}$ should be strictly positive. For a better response to external perturbations, a minimal stiffness is even imposed: $K_{\alpha}\left(\alpha_{0}, L, k_{1}, k_{2}\right)>K_{\min }$. The objective now is to determine the spring stiffnesses satisfying the minimal mechanism stiffness $K_{\min }$ at the prescribed rest configuration $\alpha_{0}$, for a given dimension $L$. Combining (11) and (9) yields :

$$
G_{b}+k_{2} X_{2}+k_{1} X_{1}=Z_{p} F_{P}
$$

where the dependency on $L$ and $\alpha_{0}$ is omitted to simplify the writting. Thus $k_{2}$ can be expressed as a function of $k_{1}, \alpha_{0}$ and $L$ :

$$
k_{2}=\frac{Z_{p} F_{P}-G_{g}-k_{1} X_{1}}{X_{2}}
$$

Using (11), (12), the condition on the mechanism stiffness writes:

$$
G_{b}^{\prime}+k_{2} X_{2}^{\prime}+k_{1} X_{1}^{\prime}-Z_{p}^{\prime} F_{P}>K_{m i n}
$$


Reporting (14) into (15) yields, after rearranging the terms:

$$
k_{1}\left[X_{1}^{\prime}-\frac{X_{1}}{X_{2}} X_{2}^{\prime}\right]>K_{m i n}+Z_{p}^{\prime} F_{P}-G_{b}^{\prime}-\frac{Z_{p} F_{P}-G_{g}}{X_{2}} X_{2}^{\prime}
$$

Since $\left[X_{1}^{\prime}-\frac{X_{1}}{X_{2}} X_{2}^{\prime}\right]>0$ the condition on $k_{1}$ can be written as $k_{1}>k_{1_{\text {min }}}$, with:

$$
k_{1_{\text {min }}}=\frac{\left(K_{\min }+Z_{p}^{\prime} F_{P}-G_{b}^{\prime}\right) X_{2}-\left(Z_{p} F_{P}-G_{b}\right) X_{2}^{\prime}}{X_{2} X_{1}^{\prime}-X_{1} X_{2}^{\prime}}
$$

Figure 3 shows the plots of $k_{1 \min }$ and $k_{2 \min }$ against the rest position $\alpha_{0}$ for several lengths $L$. Note that $k_{2 \min }$ was plotted using (14) with $k_{1}=k_{1 \min }$ to confirm the symmetric behavior. $F_{p}$ was chosen here as $F_{p}=5.9 \mathrm{~N}$, a weight that would represent the mass of about ten segments. The spring stiffness strongly depends on the link length $L$, which shows the direct influence of the geometric parameters on the mechanism stiffness $K_{\alpha}\left(\alpha, L, k_{1}, k_{2}\right)$. It is worth noting that if $\alpha_{0}$ is too large, namely if $\alpha_{0}<-\pi / 2$ or $\alpha_{0}>\pi / 2$, excessive spring stiffnesses are generally required for the mechanism minimal stiffness to be satisfied.
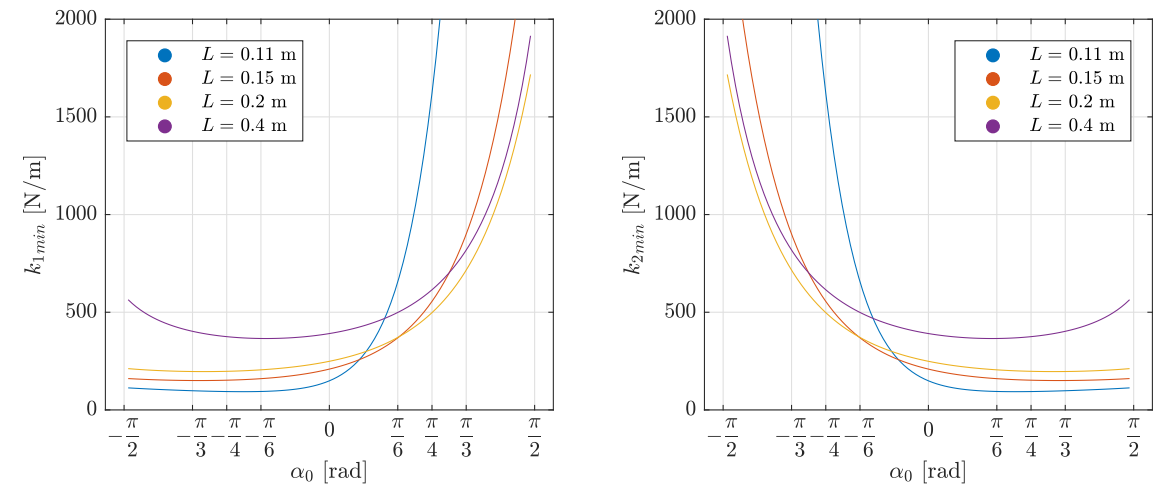

Fig. 3: Minimal spring stiffness $k_{1 \min }$ (left) and $k_{2 \min }$ (right) for different link lengths $L$, against the prescribed rest configuration $\alpha_{0}\left(K_{\min }=1 \mathrm{Nm} / \mathrm{rad}\right.$, $F_{P}=5.9 N$ and $\left.\left(\hat{x}_{P}, \hat{y}_{P}\right)=(0.05,0.1)\right)$.

\section{Description of the Wrench feasible workspace and influence of the parameters}

Using (9), and rearranging the terms, the static equilibrium condition is:

$$
G_{b}(\alpha, L)+G_{s}\left(\alpha, L, k_{1}\right)-Z_{p}(\alpha, L) F_{P}=Z_{1}(\alpha, L) F_{1}+Z_{2}(\alpha, L) F_{2}
$$


The right-hand side of (18) is the actuation wrench. The wrench feasible workspace (WFW) must satisfy, by definition [9], the geometric constraints (3), the static equilibrium (9) and the limits of the external forces $F_{\max }$ and $F_{\min }$ as introduced in Sec. 2. The coefficients $Z_{1}$ and $Z_{2}$ defined by (10a) can be shown to satisfy $Z_{1}(\alpha)>0$ and $Z_{2}(\alpha)<0$ for $-\pi<\alpha<\pi$.

Accordingly, it is possible to determine the limits of the actuation wrench as a function of the limits of the actuation forces as follows:

$$
\begin{aligned}
& \Gamma_{\max }(\alpha)=Z_{1}(\alpha) F_{\text {max }}+Z_{2}(\alpha) F_{\text {min }} \\
& \Gamma_{\min }(\alpha)=Z_{1}(\alpha) F_{\text {min }}+Z_{2}(\alpha) F_{\text {max }}
\end{aligned}
$$

Figure 4 shows the bounds of the actuation wrenches for different link lengths as defined by (19). The area enclosed by the dashed curves indicates the feasible wrench area. Several instances of $G(\alpha)=G_{b}(\alpha, L)+G_{s}\left(\alpha, L, k_{1}\right)-Z_{p}(\alpha, L) F_{P}$ are present in the figures as well. The static equilibrium equation (9) can be satisfied for a range of $\alpha$ where $G$ is within the feasible wrench bounding curves:

$$
\Gamma_{\min }(\alpha) \leq G(\alpha) \leq \Gamma_{\max }(\alpha)
$$

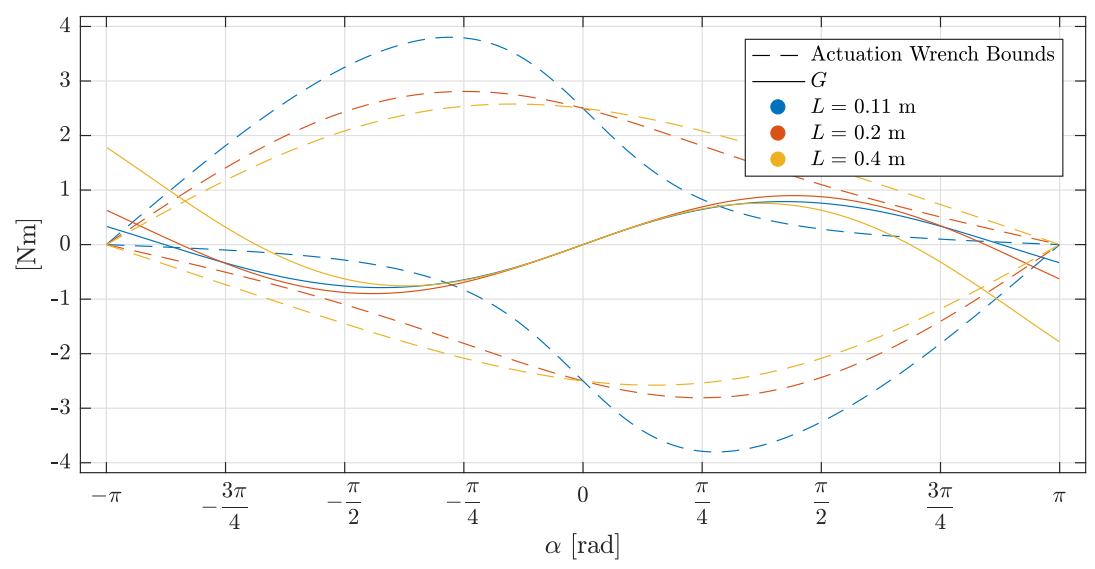

Fig. 4: Actuation wrench bounds and $G(\alpha)$ for varying link lengths $L$.

The limits of the WFW are determined by the intersections of $G(\alpha)$ with $\Gamma_{\min }$ and $\Gamma_{\max }$. If several intersections occur between $G(\alpha)$ and $\Gamma_{\min }$ or $\Gamma_{\max }$, the WFW is non-connected, meaning that it is not fully reachable in statics. Accordingly, the bounds of the WFW are taken as the first intersections that occur starting from the rest position $\alpha_{0}$. In Figure 4, three instances of $G$ were plotted for different link lengths $L$ : for each length $L$, the minimal stiffnesses $k_{1 \min }$ and $k_{2 \min }$ were computed (here for $K_{\min }=1 \mathrm{Nm} / \mathrm{rad}, F_{P}=5.9 \mathrm{~N}$, $\left(\hat{x_{P}}, \hat{y_{P}}\right)=(0.05,0.1)$ and $\left.\alpha_{0}=0\right) . \Gamma_{\min }$ and $\Gamma_{\max }$ are influenced by $L, F_{1}$ 
and $F_{2}$ (here $F_{\min }=0 \mathrm{~N}$ and $F_{\max }=50 \mathrm{~N}$ ). It is apparent that the size of the WFW depends on $L$. Here the WFW is the smallest for $L=0.11 \mathrm{~m}$, but is greater for $L=0.2 \mathrm{~m}$ than for $L=0.4 \mathrm{~m}$, which means that depending on the desired parameters $\left(\alpha_{0}, F_{P}\right.$ and its application point $\left.P\left(\hat{x}_{P}, \hat{y}_{P}\right)\right)$, an optimal value $L$ can be found in order to maximize the WFW.

Figure 5 shows the influence of the different external parameters. Prescribing a non-zero rest position $\alpha_{0}$ shifts the WFW and reduces it; decreasing $F_{P}$ increases the size of the WFW. The bounds of the WFW are also influenced by the position of $P$ with respect to the upper bar.

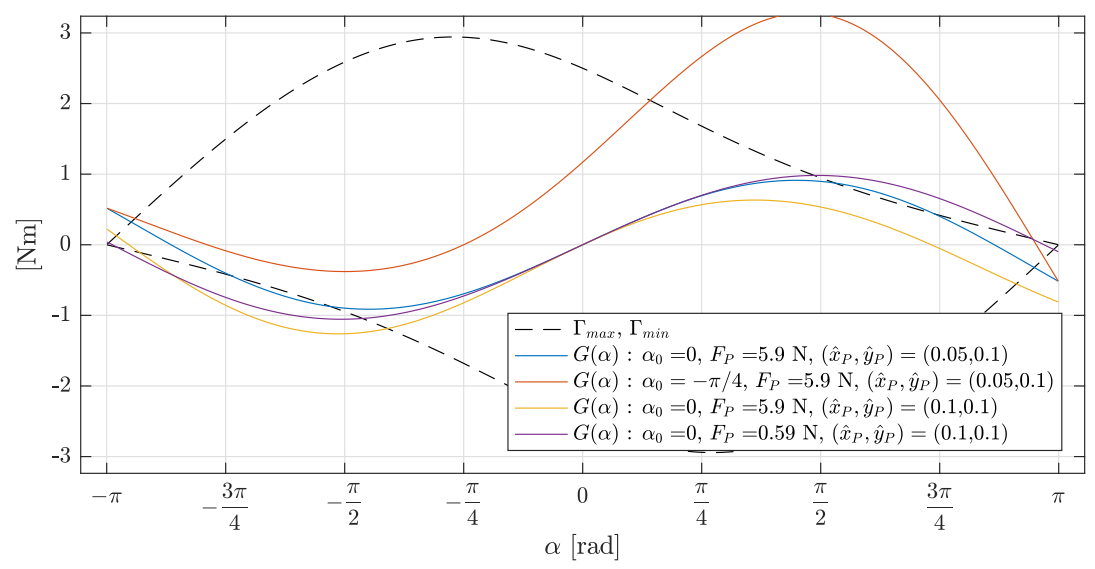

Fig. 5: Actuation wrench bounds and $G(\alpha)$ for varying desired parameters $\alpha_{0}$, $F_{P}$ and $\left(\hat{x}_{P}, \hat{y}_{P}\right)$. Blue and red (resp. blue and yellow, yellow and purple) curves $G(\alpha)$ show the effect of a change in $\alpha_{0}$ (resp. $\hat{x_{P}}, F_{P}$ ).

\section{Optimal design of the tensegrity mechanism for given specifications}

As shown in previous section, one can find a minimal stiffness for the springs depending on the link length $L$, given that the minimal segment stiffness $K_{\min }$, the application point, the force $F_{P}$ and the rest position $\alpha_{0}$ are prescribed. Since the minimal and maximal wrenches applied by the cables also depend on $L$ ( $F_{\max }$ and $F_{\min }$ are known), an optimal value of the link length $L$ can be found for the aforementioned prescribed parameters.

An algorithm has been written in order to compute the size of the WFW $W_{s}$ defined as : $W_{s}=\left|\alpha_{\max }-\alpha_{\min }\right| . \alpha_{\max }$ (resp. $\alpha_{\min }$ ) is obtained by computing the two first intersection of $G$ with $\Gamma_{\max }$ and/or $\Gamma_{\min }$ from the rest position $\alpha_{0}$. The optimal length $L$ is found when $W_{s}$ is maximal.

Figure 6 shows the size of the WFW and the optimal value of $L$ for different sets of prescribed parameters. When comparing the blue and the red curves, it 
is apparent that the optimal link length and the size of the WFW increase when $F_{P}$ decreases, which means that for higher external wrenches, the mechanism needs to be more compact and has a reduced WFW. For instance, the blue curve shows the optimal value for a rest configuration $\alpha_{0}=0$ of a segment that must carry around 10 segments above it. This value can be used to design a segment near the base of the neck. On the other hand, the red curve is computed for the same prescribed parameters but with a low external wrench, which can emphasize the case of a segment close to the bird head. When comparing the blue and the purple curves, plotted for $\alpha_{0}=0$ and $\alpha_{0}=-\pi / 4$, respectively, one can see that depending on the rest position, the optimal value of $L$ will be different. Those three curves were plotted for the minimal values of $k_{1}$ and $k_{2}$ found to satisfy (14) and (17). Nevertheless, higher stiffness values can be chosen, as illustrated by the yellow curve $\left(k_{1}=2 k_{1 \text { min }}, k_{2}=k_{1}\right.$ as $\left.\alpha_{0}=0\right)$. In this case, the size of the WFW is reduced but as the mechanism is stiffer, the optimal length value is increased. Accordingly, the minimal spring stiffness should be taken into account for the final design of the segment. It can also be observed in Figure 6 that the size of the WFW does not change significantly when $L$ is increased from its optimal value. A drastic change is observed if $L$ is diminished for large workspaces but not for small workspaces.

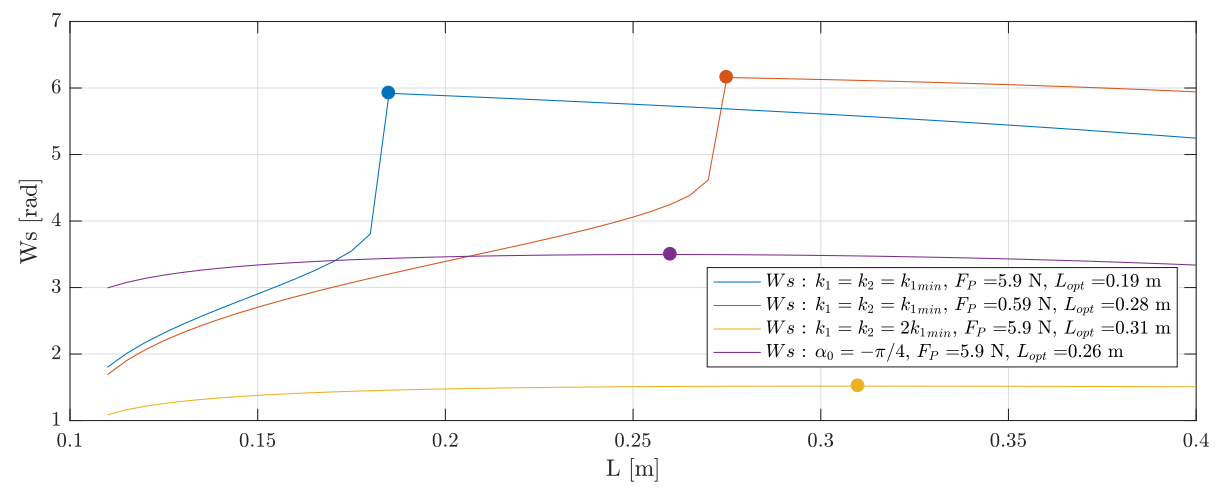

Fig. 6: Size of the WFW and optimal length $L$. For each case $K_{\min }=1 \mathrm{Nm} / \mathrm{rad}$ and $\left(\hat{x}_{P}, \hat{y}_{P}\right)=(0.05,0.1)$.

\section{Conclusion}

A methodology to design cascaded Snelson X-shape mechanisms with two lateral springs used in a bird neck model, has been proposed in this paper. The mechanisms are subject to their own weight and to an external wrench $F_{P}$. The link lengths and the spring stiffnesses of the mechanism are optimized as a function of a prescribed rest configuration with a given stiffness at rest and for a 
maximal wrench feasible workspace. The proposed methodology will be applied in an iterative scheme to design the complete S-shape bird neck model made of $n$ segments. The top segment (segment $n$ ) is first designed, where $F_{P}$ defines the head weight. Then segment $n-1$ is designed and this time $F_{P}$ integrates the weight of segment $n$ in addition to the head weight, and so on until the base segment. In this work, constant limits on the actuation forces have been assumed. The choice of the actuation scheme of the complete mechanism is still an open issue and will be decided later in the light of the muscle organization of the bird neck, which is under investigation by our biologist partners.

Acknowledgement This work was conducted with the support of the French National Research Agency (AVINECK Project ANR-16-CE33-0025).

\section{References}

1. Motro, R. Tensegrity systems: the state of the art, Int. J. of Space Structures, 7 (2), pp 75-83, 1992

2. K. Snelson, 1965, Continuous Tension, Discontinuous Compression Structures, US Patent No. 3,169,611

3. R. B. Fuller, Tensile-integrity structures, United States Patent 3063521,1962

4. Skelton, R. and de Oliveira, M., Tensegrity Systems. Springer, 2009

5. M. Arsenault and C. M. Gosselin, Kinematic, static and dynamic analysis of a planar 2-dof tensegrity mechanism, Mech. and Mach. Theory, Vol. 41(9), 1072-1089, 2006

6. C. Crane et al., Kinematic analysis of a planar tensegrity mechanism with presstressed springs, in Advances in Robot Kinematics: analysis and design, pp 419-427, J. Lenarcic and P. Wenger (Eds), Springer (2008)

7. P. Wenger and D. Chablat, Kinetostatic Analysis and Solution Classification of a Planar Tensegrity Mechanism, proc. 7th. Int. Workshop on Comp. Kinematics, Springer, ISBN 978-3-319-60867-9, pp422-431, 2017.

8. S. Levin, The tensegrity-truss as a model for spinal mechanics: biotensegrity, J. of Mechanics in Medicine and Biology, Vol. 2(3), 2002

9. Q. Boehler et al., Definition and computation of tensegrity mechanism workspace, ASME J. of Mechanisms and Robotics, Vol 7(4), 2015

10. JB Aldrich and RE Skelton, Time-energy optimal control of hyper-actuated mechanical systems with geometric path constraints, in 44th IEEE Conference on Decision and Control, pp 8246-8253, 2005

11. S. Chen and M. Arsenault, Analytical Computation of the Actuator and Cartesian Workspace Boundaries for a Planar 2-Degree-of-Freedom Translational Tensegrity Mechanism, Journal of Mech. and Rob., Vol. 4, 2012

12. D. L Bakker et al., Design of an environmentally interactive continuum manipulator, Proc.14th IFToMM World Congress in Mechanisms and Machine Science, Taipei, Taiwan, 2015

13. G. Zweers, R. Bout, and J. Heidweiller, Perception and Motor Control in Birds: An Eco- logical Approach. Springer, 1994, ISBN: 978-3-642-75869-0.

14. J.E. von Boas, Biologisch-anatomische Studien über den Hals der Vögel Andr. Fred. Host and Son.Köbenhavn, 1929

15. Q. Boehler, M. Vedrines, S. Abdelaziz, P. Poignet, P. Renaud, Design and evaluation of a novel variable stiffness spherical joint with application to MR-compatible robot design. In Robotics and Automation (ICRA), 2016 IEEE International Conference on (pp. 661-667). 